\title{
International Journal of Ayurveda and Pharma Research
}

\author{
Review Article
}

\section{APPROACH TO INTEGRATIVE MASTER HEALTH CHECK COMBINING HEALTH SCREENING PARAMETERS OF AYURVEDA AND BIOMEDICINE - A NARRATIVE REVIEW}

\author{
Vivek Sanker MK $^{1 *}$, Naidu MUR ${ }^{2}$, Gangadharan GG $^{3}$ \\ ${ }^{*}{ }_{1} \mathrm{PhD}$ Scholar, Manipal University, Manipal, Karnataka. \\ 2Formerly Dean, Nizam's Institute of Medical Science, Hyderabad, Telangana. \\ 3Medical Director, Ramaiah Indic Specialty Ayurveda Restoration Hospital (RISA), Bangalore, Karnataka, India.
}

\begin{abstract}
Health check-up is an annual program that attempts to reduce health care costs by early detection of disease symptoms before its usual clinical presentation that can encourage adoption of preventive measures or early treatment. There is a concern among clinicians that the standard tests under health screening packages could be off target in terms of detection of relevant symptoms as far as a few individual groups are concerned and end-up in wastage of money of the end customer. The article presents a review of the current evidences for and against screening of healthy volunteers enlists some of the key challenges that have been reported in health check-up programs and argues the need to give individualized advice on diet and lifestyle through suitable integration of concepts of Ayurveda. It outlines the components of an integrated health screening parameter developed from the ten-fold clinical examination procedure recommended in Ayurveda (called Dasavidhapareeksha) and argues the case for critically drawing from the strengths of both systems of medicine.
\end{abstract}

KEYWORDS: Ayurveda, Health check-up, Health screening, Preventive screening, Integrative medicine.

\section{INTRODUCTION}

Health check-up is an accepted means to disease prevention and today undergoing health check-up is integral to health-seeking behavior world over. It is argued that making people aware of risk factors for a disease will enable them to exert greater control over their own health. The Master Health Check-up (MHC) offered by various healthcare centers (like multi-specialty hospitals, diagnostic centers) is an annual program that attempts to reduce health care costs by early diagnosis and its implications for prevention of diseases. The underlying assumption behind this health check-up strategy is that a variety of chronic diseases that afflict us, most of which take their toll after the fifth decade of life, can be diagnosed at an early stage. Diabetes, hypertension, Ischemic Heart Diseases, Cerebro-vascular Stroke and certain types of Cancer are some of the more common examples. Almost all of these problems are characterized by a long quiescent phase where they produce no or little symptoms that can be as long as 10 years. It makes sense, therefore, that a health check-up program that attempts to detect and correct these problems during this silent phase will decrease the ultimate morbidity and mortality arising from these diseases.

\section{Health Check in Biomedicine}

Tests done under a standard health package are deemed to be selected with sufficient merit. They can help assess the total health of an individual, diagnose silent diseases such as hypertension or diabetes and even detect early complications. At the level of an individual, these tests also offer a sense of health security achieved by undergoing these tests and discovering them to be negative.

There is however a concern among clinicians especially in the developed countries that the standard tests that are performed at labs as part of health screening packages could be off target in many cases as had emerged in several studies on the utility of health screening measures as an effective preventive strategy. ${ }^{[1-4]}$ It is felt that these test results may sometimes confuse the attempt to trace the real problem and all the tests may not be relevant for a particular individual and end in wastage of money and valuable resources. In some studies it even emerged, although not conclusively, that indiscriminate screening of healthy individuals may even have an adverse impact on health. ${ }^{[5,6]}$ In this review, done as part of an effort to develop a tool for Integrative Master Health Check by combining 
modalities of Bio-medicine and Ayurveda, we present the current evidences for and against screening of healthy volunteers and argue the case for critically drawing from the strengths of both systems of medicine (Ayurveda and Bio-medical science) to make the screening of healthy volunteers more meaningful and effective.

The review covers articles searched from the PUBMED database using the key words 'Health Screening', 'Health Checks' and 'Health Check-up'. Among relevant articles, few argue for and many against a health screening program. We have attempted to present the relevant one's among these in order to provide the reader with an appreciation of the current level of evidence for preventive screening and also the fact that there is a clear lack of evidence for the effectiveness of random tests performed as part of conventional health checks in hospitals and diagnostic centers in preventing chronic diseases.

\section{Evidence against screening of Healthy Volunteers}

One of the early evidence against screening of healthy volunteers was provided by the Southeast London Screening Study. This study was as a longterm controlled trial of multiphasic screening directed at middle-aged individuals registered with their family doctors in the year 1967, and involved 7,229 subjects, aged 40 to 64 years, who were randomly allocated into either a screening or control group. The screening group was invited to attend two screening sessions held about two years apart while the control group continued to receive conventional medical care. Both groups were then invited to participate in a health survey in 1972 to 1973. Screening-control comparisons revealed no significant differences in either mortality or morbidity over the first nine years of the study with one exception: the screened males were significantly less "anxious" (as measured by the Middlesex Hospital Questionnaire) than the controls (P less than $0.01)$. In the context of other negative psychiatric findings, however, the importance of this result is considered doubtful. Despite methodological limitations, the study concluded that multiphasic screening of the middle-aged in general practice is probably not worthwhile. One study, published in the Archives of Internal Medicine argues that Annual Physical Examination, an important feature of healthcare delivery in the United States is not necessary for conveying messages on preventing illness and also costs too much. It reasons that patients get most messages on prevention through other visits. In this study, researchers at the University Of Pittsburgh Medical Center examined data from 2002, 2003, and 2004 from the US national ambulatory medical care survey and the national hospital ambulatory medical care survey, which record visits made by patients with health insurance to office based physicians and to hospital outpatient departments for annual check-ups and gynecological examinations. 'During 2002-4 about 44 million US adults had an annual preventive physical check-up and about 19 million women had a preventive gynecological examination each year. Many tests are unnecessary and in total cost more than $\$ 350 \mathrm{~m}$ a year, say the authors..$^{[7]}$

A study by Gans JE et al suggests that preventive interventions would have to eliminate $15 \%$ of adolescent morbidities overall to break even in economic terms. ${ }^{[8]}$ Studies have also suggested that there is a risk of causing psychological distress by screening healthy adults. $\left.{ }^{6]}\right]$

A simulation study compared the effectiveness of two approaches to reducing Incidence Rate of CVD in a sample population: a traditional approach, in which high-risk patients are treated with conventional antihypertensive medications, and a population-based approach, in which subjects participate in a health promotion program. The success rate-oriented simulation suggests that prescribing antihypertensive medications is superior to promoting the health promotion program in reducing IR-Cardio-Vascular Disease in virtual middle-aged Japanese men. ${ }^{[9]}$

Critiques of the Screening Industry also argue that the ability of Standard Health Screening to offer individual specific health care advice covering various domains of health like psychological, emotional and spiritual well-being is rather limited.

\section{Evidence for Screening of Healthy Volunteers}

On the other hand, an analysis of the German Kiggs survey was reported to have shown compelling evidence that scoliosis and thyroid disorders, in particular, are under diagnosed if a J1 check-up (a preventive health screening) is not performed. It was reported that if a J1 check-up were performed in the nearly two-thirds of all adolescents who currently do not undergo one, many latent health problems could be recognized and treated in timely fashion. ${ }^{[10]}$

There is a study that found that BMI was a significant parameter independently correlated with Chronic Kidney Disease in both genders. Thus, increased BMI is associated with CKD independently of blood pressure, serum lipid and glucose levels in the general population. ${ }^{[11]}$

It was reported that modeling work by the Department of Health in UK indicates that the health checks will prevent at least 1600 heart attacks and strokes and save an estimated 650 lives each year. The checks will prevent a further 4000 people from developing diabetes, the department says, and 20000 
Vivek Sanker MK et al. Integrative Master Health Check Combining Health Screening Parameters of Ayurveda and Biomedicine

people will have diabetes and kidney disease detected a year earlier, allowing for better management.[12]

\section{Evidences recommending modifications in exiting screening protocols}

Routine assessment of global coronary heart disease (CHD) risk scores are recommended by Standard Guidelines. A systematic review was performed to assess whether global CHD risk scores result in clinical benefits or harms. The study concluded that preliminary evidence suggest that physicians knowledge of global CHD risk scores may translate into modestly increased prescribing of cardiovascular drugs and modest short-term reductions in CHD risk factors without clinical harm. However, it states that whether these results are replicable, and translate across other practice settings or into improved long-term CHD outcomes remains to be seen.[13]

A study by Theresa MM and Ann LK published in BMJ contended that by presenting the uncertainties associated with the assessment and reduction of cardiovascular risk has the potential to be more cost effective than screening conducted in a traditional, Public health paradigm if it results in participants who are more motivated to reduce their risks. It also has the potential to increase inequalities by reducing the number of people most at risk who participate in screening. Evaluation of the operation of an informed choice approach to cardiovascular risk assessment is needed.[14] A study by Stacy et al found that global CHD risk information seems to improve the accuracy of risk perception and may increase intent to initiate CHD prevention among individuals at moderate to high risk. The effect of global risk presentation on more distal outcomes is less clear and seems to be related to the intensity of accompanying interventions.[15]

\section{Challenges in Screening of Healthy Volunteers}

Some of the key challenges that have been reported in health check-up programs are:

1. Conclusive evidence of effectiveness and cost effectiveness is yet to emerge.

2. Uptake of such programs is low in weaker socioeconomic and multiethnic communities

3. Interventions to increase physical activity have had limited success

4. Implementation is likely to be challenging in an already overstretched primary care delivery system

5. Information technology to capture and transfer data between organizations providing health checks is not yet available.[16]

\section{DISCUSSION}

\section{Emerging health scenario}

It is important at this juncture to look at the emerging global health scenario to fundamentally understand what is it that an ideal preventive strategy should aim to achieve specifically through screening of healthy adults. The World Health Organization predicts that Non-communicable conditions will cause over three quarter of all deaths in 2030. Globally, death from cancer will increase from $7.4 \mathrm{M}$ in 2004 to $11.8 \mathrm{M}$ in 2030 , and deaths from cardio-vascular will rise from $17.1 \mathrm{M}$ to $23.4 \mathrm{M}$ in the same period. By 2030, deaths due to cancer will collectively account for $56 \%$ of the predicted 67 $M$ deaths due to all causes.[17] In the context of a large country like India, where the state machinery is severely hard-pressed to meet the ever increasing healthcare needs of a large section of its population that cannot afford private healthcare, it becomes all the more important to develop suitable cost-effective strategies to address the increasing burden of disease and its impact on productivity. Annual income loss due to illness and care giving was estimated by one study to be US\$23 billion for 2004 from all noncommunicable diseases. ${ }^{[18]}$

There is a clear and evident change in the disease burden which calls for an appropriate paradigm shift in the health care delivery systems as well. Given this, it would not be an overstatement to say that the $21^{\text {st }}$ Century will be the era of pluralism in Health Care as the human population, the world over, is not getting viable solutions for all their health needs in any one single medical system. Therefore the search and growing acceptance of complementary systems of health care.

\section{Health Check in Ayurveda}

Ayurveda is an evidence based health science (Pramana shastra) that has evolved and matured over the last two millennia. It therefore has the potential to be a significant contributor in the global search for complementary health care. The complete examination of Svasthya (a healthy individual) to assess the different components of health was in practice during classical age. Ayurveda enlists a set of tools (subjective) to assess the health of an individual (the Dasavidha pareeksha (ten-fold examination), $[19,20]$ being the most comprehensive of them) and advocates elaborate individual specific dietary and lifestyle regimens that covers various domains of health like physical, psychological, emotional and spiritual.

The idea of an Integrative Master Health Check-up (IMHC) is to make it possible to find the weakness in different domains of health and person's susceptibility to diseases. It then aims to prevent the 
occurrence of diseases by managing, through suitable life style changes, stress management, detox therapy (Panchakarma) and Rejuvenative therapy (Rasayana). The central idea in IMHC is a ten-fold clinical assessment of humans advised in Ayurveda known as the Dasavidha pareksha. This includes body constitution (Prakriti), pathological state (Vikriti), tissue vitality (Sara), physical build (Samhanana), Anthropometry (Pramana), adaptability (Samya), psychic constitution (Satva), capacities for digestion (Ahara-Shakti), exercise tolerance (Vyayama-shakti) and age (Vaya).

The ten-fold examination (Dasavidha pareeksha) was then, for better comprehension, divided into three domains namely physical, psychological and metabolic (Agni). One more domain i.e. immunological (Vyadhikshamatva) was added in order to assess immune response separately even though all the other 3 domains contribute and constitute it. Assessing these domains individually helps in identifying the weaknesses of each of the domain allowing one to advice appropriate promotive and preventive measures.

Hence the Ayurveda component of IMHC had four domains namely, Domain I - Shareera (Physical): Includes Dasavidhapareeksha excluding Vikriti, Satva and Agni pareeksha. Domain II- Manasa (Psychological): Includes Satva pareeksha. Domain III - Agni (Metabolic): Includes Ahara Shakti and Agni pareeksha. Domain IV- Vyadhi Kshematva (Immunological).

However, the evidence of its effectiveness available is disciplinary (rooted in Ayurvedic discipline) and not 'trans-disciplinary'. Generating trans-disciplinary evidence and correlations is a complex task because it requires assimilation of the disciplinary knowledge of Ayurveda (which relies on systems based theories like the three humor) and finding its correlation with western bio-medicine (which relies on structural theories).

\section{Need for an Integrative Approach in Health Checks}

It is evident, from various studies quoted above demonstrating serious limitations in health screening programs, that we need to look at broadening the scope of a standard health check-up to give individual specific advice on diet and lifestyle that can achieved through suitable integration of certain concepts of Ayurveda like an individual's constitutional make-up (Prakruti), metabolic makeup (Agni) etc.

Figure 1: The comprehensive health screening parameter given in Ayurveda

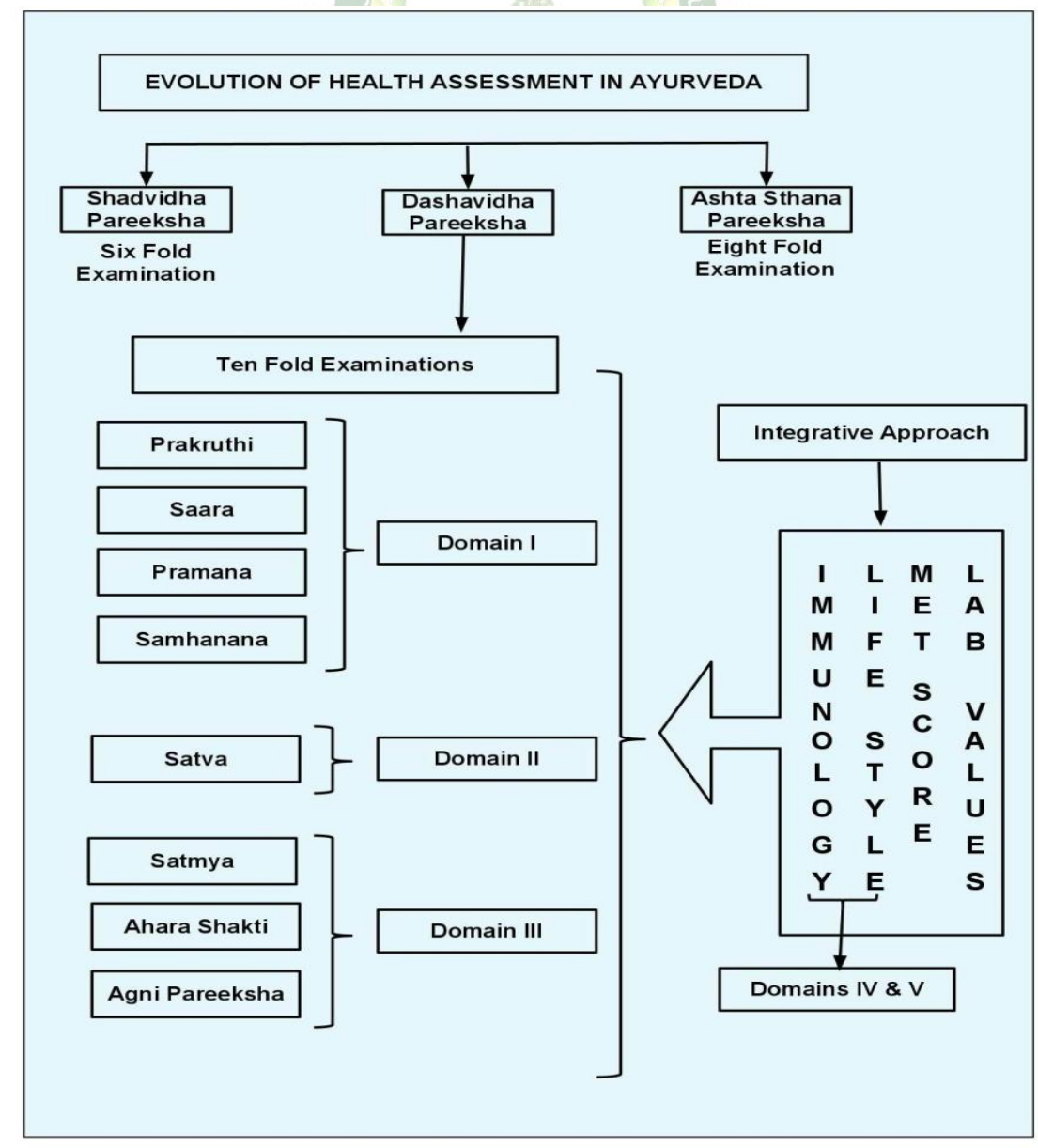




\section{CONCLUSION}

It may be argued that a comprehensive integrative health check-up strategy that combines conventional tests with Ayurveda will address gaps that exist and present a desirable objective that needs to be actively pursued.

\section{REFERENCES}

1. A controlled trial of multiphasic screening in middle-age: results of the South-East London Screening Study. The South-East London Screening Study Group. Int J Epidemiol. 1977; 6(4):357-363.

2. Collen MF, Dales LG, Friedman GD, Flagle CD, Feldman R, Siegelaub AB. Multiphasic checkup evaluation study. 4. Preliminary cost benefit analysis for middle-aged men. Prev Med. 1973; 2(2): 236-246.

3. Multiple risk factor intervention trial. Risk factor changes and mortality results. Multiple Risk Factor Intervention Trial Research Group. JAMA. 1982;248 (12):1465-1477.

4. Rose G, Tunstall- Pedoe HD, Heller RF. UK heart disease prevention project: incidence and mortality results. Lancet. 1983; 1(8333):1062-1066.

5. Haynes RB, Sackett DL, Taylor DW, Gibson ES, Johnson AL. Increased absenteeism from work after detection and labeling of hypertensive patients. $\mathrm{N}$ Engl J Med. 1978; 299(14):741-744.

6. Stoate HG. Can health screening damage your health?. J R Coll Gen Pract. 1989; 39(322):193-195.

7. Mehrotra A, Zaslavsky AM, Ayanian JZ. Preventive health examinations and preventive gynecological examinations in the United States. Arch Intern Med. 2007; 167(17):1876-1883.

8. Gans JE, Alexander B, Chu RC, Elster AB. The cost of comprehensive preventive medical services for adolescents. Arch Pediatr Adolesc Med. 1995; 149(11):1226-1234.

9. Ohno Y, Shibazaki S, Araki R, et al. Antihypertensive medication versus health promotion for improving metabolic syndrome in preventing cardiovascular events: a success rate-oriented simulation study. BMC Med Inform DecisMak. 2011; 11:8. Published 2011 Feb 13.

10. Hagen B, Strauch S. The J1 adolescent health checkup: analysis of data from the German KiGGS survey. Dtsch Arztebl Int. 2011; 108(11):180-186.

\footnotetext{
Cite this article as:

Vivek Sanker MK, Naidu MUR, Gangadharan GG. Approach To Integrative Master Health Check Combining Health Screening Parameters of Ayurveda And Biomedicine - A Narrative Review. International Journal of Ayurveda and Pharma Research. 2020;8(Suppl 1):77-81.

Source of support: Nil, Conflict of interest: None Declared
} 60 230-239. bmj.c6312 World Bank.
11. Nomura I, Kato J, Kitamura K. Association between body mass index and chronic kidney disease: a population-based, cross-sectional study of a Japanese community. Vasc Health Risk Manag. 2009;5(1):315-320.

12. Kmietowicz, Zosia. Five Yearly Checks for Over $40 \mathrm{~s}$ Will Save 650 Lives a Year, Says Government. BMJ: British Medical Journal (Online), vol. 338, 2009. doi:http://dx.doi.org/10.1136/bmj.b1334.

13. Sheridan SL, Crespo E. Does the routine use of global coronary heart disease risk scores translate into clinical benefits or harms? A systematic review of the literature. BMC Health Serv Res. 2008; 8:60. Published 2008 Mar 20. doi:10.1186/1472-6963-8-

14. Marteau TM, Kinmonth AL. Screening for cardiovascular risk: public health imperative or matter for individual informed choice. BMJ. 2002; 325(7355):78-80.

15. Sheridan SL, Viera AJ, Krantz MJ, et al. The effect of giving global coronary risk information to adults: a systematic review. Arch Intern Med. 2010;170(3):

16. Khunti K, Walker N, Sattar N, Davies M. Unanswered questions over NHS health checks. BMJ. 2010; 342:c6312. Published 2010 Jan 26. doi:10.1136/

17. C Mathers, DM Fat, JT Boerma, WHO. The Global Burden of Disease: 2004 Update, World Health Organization (2008)

18. Mahal A, Karan, A, Engelgau MM. 2010. -The Economic Implications of Non Communicable Diseases for India.\| Health, Nutrition, and Population Discussion Paper. Washington DC,

19. Acharya charaka, Acharya Drdhabala, Acharya Chakrapanidatta. The Charaka samhita of Agnivesha. 5 ed. Varanasi: Munshiram Manoharlal Publishers Pvt. Ltd, 1992.

20. Acharya vagbhata, Acharya Arunadatta, Acharya hemadri. Ashtangahridaya. Varanasi: Krishnadas Academy, 1995

*Address for correspondence
Dr MK Vivek Sanker
\#205, Kempton Kourt
Apartments
Site 317 Duo Marvel Layout,
Yelahanka, Bengaluru 560064
Karnataka, India
Email: dr.mkvivek@gmail.com
Mobile: +91-7093320771

Disclaimer: IJAPR is solely owned by Mahadev Publications - dedicated to publish quality research, while every effort has been taken to verify the accuracy of the content published in our Journal. IJAPR cannot accept any responsibility or liability for the articles content which are published. The views expressed in articles by our contributing authors are not necessarily those of IJAPR editor or editorial board members. 